\title{
CREACIÓN DE UN METAVERSO EN OPENSIM PARA LA UNIVERSIDAD DISTRITAL DENTRO DE LA RED RITA-UD
}

\section{THE MAKING OF AN OPENSIM METAVERSE FOR UNI- VERSIDAD DISTRITAL WITHIN THE RITA-UD NET- WORK}

\begin{abstract}
This article explains the setup process of a wireless network segment intended to operate in conjunction with network RITA-UD. The purpose of the segment is to provide multimedia-content access through an OpenSim-created virtual world. The streamingconstituent contents are stored in a server that is located at CECAD (a High Performance Computation Center). Together with multicast-streaming tests, transmission data were captured using WireShark so as to determine QoS parameters that allow channel characterization.
\end{abstract}

Key words: virtual environments, metaverse, OPENSIM.

\section{RESUMEN}

Este paper contempla el montaje de un segmento de red inalámbrico para la red RITA-UD con el fin de brindar acceso a contenidos multimedia a través de un mundo virtual creado en OPENSIM. Los contenidos de las emisiones de streaming se encuentran almacenados en un servidor ubicado en el centro de computación de alto desempeño, CECAD. Al tiempo que se realizaron pruebas de streaming multicast, se capturaron dichas transmisiones mediante el sniffer de paquetes WireShark con el fin de determinar parámetros de QoS que caractericen el canal.

Palabras claves: ambientes virtuales, metaverso, OPENSIM.

\section{Nicolás Torres Vallejo}

Ing. Electrónico Universidad Distrital Francisco José de Caldas, pertenece al grupo de investigación LIDER. Bogotá, Colombia.

nickolaz88@gmail.com

\section{Edward Johannes Uribe Sierra}

Ing. Electrónico Universidad Distrital Francisco José de Caldas, pertenece al grupo de investigación LIDER. Bogotá, Colombia.

edwannes@gmail.com

\section{Roberto Ferro Escobar}

Ing. Electrónico, MSc. en Teleinformática, docente de planta de la Universidad Distrital "Francisco José de Caldas". Bogotá, Colombia. rferro@udistrital.edu.co

Tipo: Artículo de revisión

Fecha de Recepción: Septiembre 5 de 2012 Fecha de Aceptación: Noviembre 1 de 2012 


\section{INTRODUCCIÓN}

El presente paper presenta a continuación reúne algunos componentes del trabajo que está realizando el grupo de investigación LIDER, acerca de la implementación de una plataforma para servicios Streaming [1] y repositorio digital en la red RITA-UD [2]. En la actualidad, la Universidad Distrital Francisco José de Caldas se enfrenta a la necesidad de implementar segmentos de red funcionales y dar cobertura con la red RITA-UD a lo largo de todas las facultades.

Además es de carácter prioritario generar servicios y contenidos para dicha red, toda vez que en su condición de red académica, debe ser de utilidad para la comunidad universitaria. Actualmente la red RITA-UD se encuentra en etapa de pruebas y se han desarrollado pocos proyectos relacionados con esta, desperdiciando así un gran potencial tecnológico [3], que en un futuro, por medio de la conexión con infraestructuras como la del CECAD o la sala ACCESS-GRID, pueden permitir a la comunidad universitaria posicionarse como centro de generación de conocimiento y ejemplo en el uso de las nuevas tecnologías. En ese orden de ideas es necesaria la gestión de proyectos que estén orientados al desarrollo de esta red como una herramienta realmente útil y necesaria para la academia, con funciones definidas y resultados tangibles [4].

Por ser de alta velocidad, la red RITA-UD es idónea para la transmisión de grandes flujos de datos, como lo requiere el servicio de streaming de video o audio [5]. La implicación social del proyecto radica en el gran impacto que tienen las redes de datos dedicadas al apoyo y soporte de la investigación dentro de las comunidades universitarias [6], ayudando a la divulgación de resultados y mejorando la disponibilidad de estos para estudiantes y profesores. La información que se verá a continuación aborda la descripción de los servicios que se implementaron para la red RITA-UD y la implementación del segmento inalámbrico de la misma, con el objetivo de ayudar a los procesos investigativos de la universidad [7].

\section{MARCO TEÓRICO}

A continuación se explican brevemente las herramientas y paquetes que se utilizaron durante el desarrollo del trabajo. Como se verá, se emplearon básicamente tres herramientas, que en conjunto permitieron que el ambiente virtual fuera funcional.

\subsection{Open simulator}

OPENSIM es un servidor de aplicaciones 3D que utiliza los mismos estándares de SecondLife para comunicarse con sus usuarios. Surge a partir del análisis de la estructura del código fuente del visor de SecondLife, liberado a principios de 2007 por Linden Labs bajo licencia GPL, aplicando ingeniería inversa con el fin de crear un servidor de aplicaciones 3D. Se caracteriza por estar basado Software Libre, poseer una estructura modular escrita en C\# y soportar múltiples visores; a la fecha OPENSIM se encuentra en la versión 0,7 [8]. Uno de los mayores potenciales de OPENSIM es la facilidad con la que los centros de educación pueden personalizar sus desarrollos, diseñar sistemas de administración e integrar usuarios de las bases de datos de sus LMS (Learning Management Systems) con la base de datos de OPENSIM.

\subsubsection{Moodle}

Moodle (siglas en inglés de "Entorno Modular de Aprendizaje Dinámico Orientado a Objetos") es un paquete de software para la creación de cursos y sitios web basados en internet de código abierto desarrollado en PHP y diseñado para trabajar sobre diferentes motores de bases de datos (MySql), que tiene como objetivo ayudar a educadores a crear y gestionar de manera sencilla comunidades de aprendizaje en línea. Moodle está pensado para propiciar la construcción del conocimiento desde el estudiante.

\subsubsection{Sloodle}

El proyecto SLOODLE (Simulation Linked Object Oriented Dynamic Learning Environment) es un proyecto de código abierto que integra los ambientes virtuales multiusuario como Second 
Life y OPENSIM con la plataforma de gestión de aprendizaje MOODLE. Sloodle provee un amplio rango de herramientas que permiten a los mundos virtuales el aprendizaje y la enseñanza inmersiva; herramientas que están totalmente integradas con un sistema de gestión enseñanza basado en la Web utilizado por miles de educadores y estudiantes en todo el mundo.

\section{MATERIALES Y MÉTODOS}

La metodología para la implementación propuesta cumplió las fases que se explican a continuación siguiendo el modelo propuesto por Roberto Hernández Sampieri [9].

\subsection{Exploración}

La fase de exploración consistió en investigar sobre mundos virtuales, considerar casos de éxito en escenarios similares a los de la Universidad, ingresar a distintos metaversos, visitar islas de Second Life, ingresar a grillas de OPENSIM, obtener información sobre media streaming en ambientes virtualizados y consolidar una idea clara sobre el funcionamiento de estos. Luego de esto, se procedió a elaborar un prototipo para comprender y entender los fundamentos de diseño en 3D. Como punto de partida se creó un aula que contenía las herramientas que permite usar Sloodle como cabinas de registro, Quiz, glosarios y Presenter, vinculados a contenidos de prueba como imágenes, videos, presentaciones, páginas Web, alojados en un servidor con la plataforma Moodle de prueba como se observa en la Figura 1.

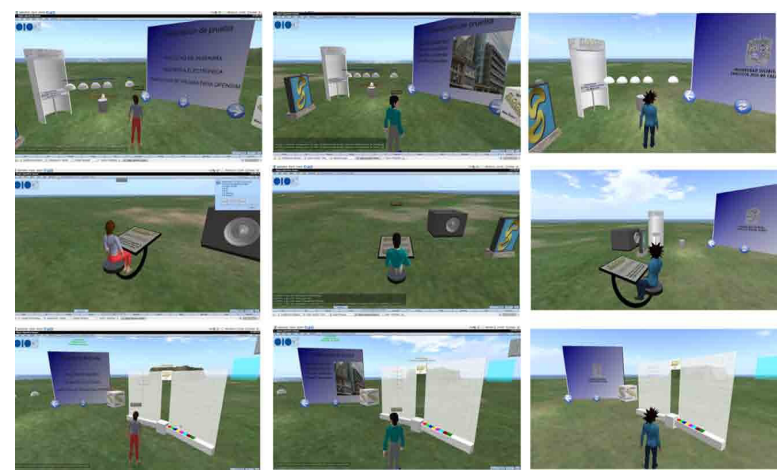

Figura 1. Contenidos de Moodle dentro de OPENSIM.

\subsection{Fase de desarrollo}

Esta fase está compuesta por la creación de los espacios que conforman el metaverso y la creación del repositorio de video streaming. Además se implementó el segmento de red inalámbrico para irradiar la red RITA-UD. Durante el desarrollo del proyecto se realizó un proceso cuidadoso de documentación con el fin de dejar plasmados todos los procedimientos.

Luego de analizar las posibles tecnologías para la implementación de un enlace y contemplar las ventajas y desventajas de cada una frente a la infraestructura del campus de la universidad, se optó por un enlace híbrido, con una parte cableada y otra inalámbrica. Cuando se tuvo funcionalidad en el canal, se realizaron emisiones de streaming de video en multicast IPv4 e IPv6, este flujo de datos fue monitoreado mediante WireShark para tomar los datos de las transmisiones y analizarlos con el fin de modelar y estimar el comportamiento del canal.

\section{MONTAJE DEL METAVERSO}

El montaje del metaverso se explica a continuación a grandes rasgos. Para alcanzar este objetivo, se contemplaron varios ítems, como el montaje del servidor Web XAMPP, la instalación de OPENSIM, la construcción de los objetos del mundo virtual, la vinculación de Moodle con los objetos y la configuración de estos para permitir el acceso a contenidos.

\subsection{Montaje de OPENSIM}

Es recomendable realizar el montaje del servidor en una versión de Linux de 32 bits, ya que las versiones de 64 bits suelen presentar problemas de compatibilidad con OPENSIM y sus dependencias. OPENSIM está desarrollado para correr sobre la plataforma .NET de Microsoft ya que gran parte de su código así como los scripts que se utilizan en los metaversos están basados en C\#, por lo que en Linux se debe instalar la plataforma Mono, que dispone de las librerías necesarias para compilar este tipo de lenguajes. Luego de la instalación se procede a correr OPENSIM, si es la primera vez se corre 
en el ordenador, no se tendrá ningún metaverso creado para iniciar, por lo que el simulador solicitara la creación de uno, pidiendo datos como el nombre de la región, identificador de región, localización de la región, máscara wilcard de usuarios admitidos, puerto interno del servidor, dirección IP del servidor y el hostname. Con lo anterior se tiene ya una región, un estado y un usuario, lo que posibilita correr el servidor y se puede acceder desde cualquier visor.

\subsection{Construcción de objetos en OPENSIM}

La creación y modificación de objetos en OPENSIM es la base para la construcción de metaversos. En la figura 2 se puede observar el visor de OPENSIM y el menú de herramientas básicas.

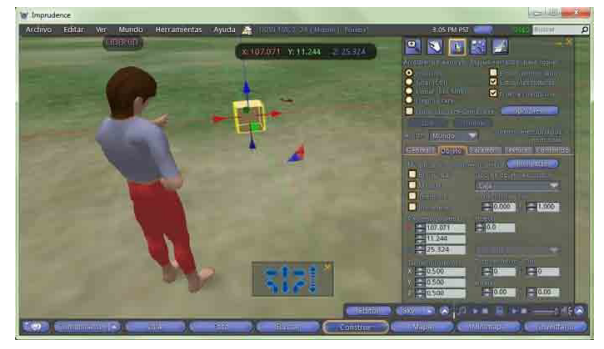

Figura 2. Visor de OPENSIM.

$\mathrm{Al}$ abrir cualquiera de las herramientas que se tienen en la parte inferior de la pantalla, se dispone de diferentes pestañas desde las cuales se pueden hacer modificaciones puntuales de los objetos creados. Entre las pestañas disponibles en el menú de herramientas encontramos las fichas general, objeto, características, textura, entre otras. Si se tiene una plataforma Moodle instalada en algún servidor accesible desde el cliente, esta puede ser vinculada al metaverso creado en OPENSIM por medio de la herramienta Sloodle de tal forma que se puedan utilizar sus funciones directamente en el metaverso. En la figura 3 se observa la interfaz de administración web de Moodle con el controlador Sloodle.

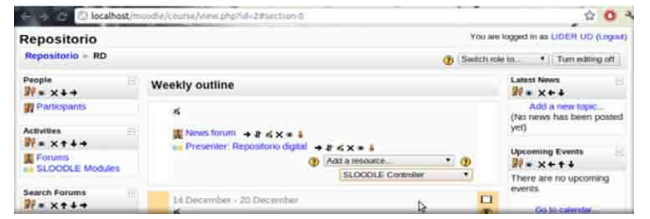

Figura 3. Interfaz de administración Web de Moodle.

\subsection{Moodle en OPENSIM}

Antes de poder acceder a Moodle desde OPENSIM, se debe activar el controlador de SLOODLE. Este controlador maneja todos los enlaces entre Moodle y OPENSIM y por lo tanto es necesario para poder utilizar cualquiera de las otras herramientas de Sloodle. Teniendo vinculados los objetos de OPENSIM, estos se usarán para acceder a los contenidos multimedia y de Streaming de video.

\section{IMPLEMENTACIÓN DEL ENLACE}

Para el segmento de red entre la oficina del grupo LIDER ubicada en el sótano 3 del edificio de ingenierías y el centro de computación de alto desempeño ubicado en el edificio sede central se hizo uso de un Access Point Cisco AP 541 conectado al SwitchCore 2950 del CECAD. La red inalámbrica radiada por el Access Point es repetida por 2 antenas NanoStation configuradas como AP repetidores llevando el acceso a RITA de forma inalámbrica hasta el auditorio sabio caldas y finalmente al grupo LIDER a través de un Router LinkSys. Desde este segmento inalámbrico de RITA se accede al repositorio de VideoStreaming y se abre la posibilidad de trabajar en radiar RITA para toda la facultad de ingeniería y las principales sedes de la universidad distrital. Las antenas NanoStation debían ubicarse a una altura que favoreciera el enlace, donde no tuvieran fácil acceso de personal no autorizado a su manipulación; debían tener línea de vista para que la potencia de la señal fuera la indicada y además cada una debía tener una posición estratégica para el enlace. La NS1 debía estar cerca de CECAD mientras que la NS2 debía estar apuntando al sótano donde se ubicaría el Router Linksys que daría conectividad al grupo LIDER.

Inicialmente se planeó trabajar inalámbricamente desde CECAD hasta LIDER, sin embargo haciendo mediciones previas, se obtuvo que la señal recibida por la primera estación era demasiado débil debido a los obstáculos existentes entre el Access Point ubicado en CECAD y la primera antena, y por lo tanto el ancho de banda no era el adecuado. Por lo anterior se pro- 
puso remplazar el primer segmento inalámbrico por uno cableado. La anterior solución se implementó habilitando un punto de red en el Switch 2950, ubicado también en CECAD y cableando un tramo de aproximadamente $30 \mathrm{~m}$ con cable CAT 6 hasta la antena para asegurar un mejor ancho de banda.

El switch cisco 2950 tiene una capacidad de procesamiento de 13,6 Gbps, con lo cual su versión de 48 puertos (la que se tiene en el CECAD) con una velocidad mínima teórica aproximada de 290 Mbps por interfaz Gigabit Ethernet o una velocidad de cable para todas sus interfaces en FastEthernet. Sin embargo, esta velocidad para los estándares se ve reducida por encabezados, acuses de recibo (en ciertos protocolos), velocidad de las interfaces de los host, capacidades de procesamiento y memoria etc. El Throughput para Gigabit Ethernet puede variar bastante, sin embargo para este caso no es relevante teniendo en cuenta que el resto del enlace limita la velocidad del canal. Típicamente para fast ethernet este valor es de $80 \mathrm{Mbps}$. Las antenas nanostation 2 también están limitadas por el estándar $802.11 \mathrm{~g}$ que ofrece aproximadamente $23 \mathrm{Mbps}$. Así, los valores máximos entre puntos del enlace teniendo en cuenta la velocidad y el Throughput especificados por los estándares
[10] se muestran en la Figura 4.

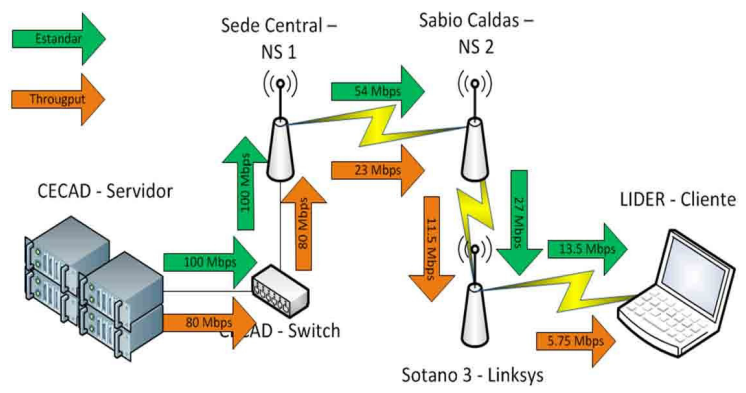

Figura 4. Enlace entre CECAD y el grupo LIDER.

Se observó que la red RITA no tiene una infraestructura ni un direccionamiento de red definido. Además de esto se encontró que anteriores proyectos no han estado enmarcados dentro de un segmento de red específico dentro de la red académica y del CECAD. Por lo anterior se propone la implementación de una red con direccionamiento IPv4 10.0.0.0/8 como prueba piloto para la organización de la red, con miras a implementar de la misma manera el direccionamiento IPv6 asignado por la red RUMBO. El diseño del segmento de red y su organización no hace parte de los objetivos de este proyecto, sin embargo se propone y se asume un esquema tentativo de red jerárquica[11][12] con las características que se definen en la tabla 1.

Tabla 1. Direccionamiento IPv4 e IPv6 para la red RITA-UD.

\begin{tabular}{|c|c|c|c|c|c|c|}
\hline & $\begin{array}{c}\text { Nombre } \\
\text { campo }\end{array}$ & $\begin{array}{c}\text { Prefijo de } \\
\text { red raíz }\end{array}$ & Facultad & $\begin{array}{c}\text { Tipo } \\
\text { acceso }\end{array}$ & $\begin{array}{c}\text { Grupo } \\
\text { final }\end{array}$ & $\begin{array}{c}\text { Dispositivo } \\
\text { (Interfaz) }\end{array}$ \\
\hline \multirow{2}{*}{$\begin{array}{c}\text { IPv4 } \\
\text { 10.0.0.0 }\end{array}$} & $\begin{array}{c}\text { Tamaño } \\
\text { campo }\end{array}$ & 8 bits & 6 bits & 2 bits & 6 bits & 10 bits \\
\cline { 2 - 7 } & Prefijo & $/ 8$ & $/ 14$ & $/ 16$ & $/ 22$ & $/ 32$ \\
\hline \multirow{2}{*}{$\begin{array}{c}\text { IPv6 } \\
2001: 13 F 8: 1150\end{array}$} & $\begin{array}{c}\text { Tamaño } \\
\text { campo }\end{array}$ & 44 bits & 6 bits & 2 bits & 6 bits & 70 bits \\
\cline { 2 - 7 } & Prefijo & $/ 44$ & $/ 50$ & $/ 52$ & $/ 58$ & $/ 128$ \\
\hline
\end{tabular}

\section{MONITOREO DE TRÁFICO CON WIRESHARK}

Wireshark es un analizador de protocolos de gran reconocimiento en el monitoreo de redes. Mediante esta herramienta se realizó el monitoreo de los paquetes transmitidos desde el servidor de streaming hacia los clientes de estas transmisiones [13]. Para la realización de todas las pruebas se utilizó el mismo procedimiento:

- Configurar wireshark e iniciar la captura de los paquetes de interés.

- Iniciar la herramienta que permita la alteración del tráfico con el fin de crear los diferentes escenarios.

- Configurar VLC con los parámetros reque- 
ridos para las diferentes condiciones.

- Detener captura paquetes con wireshark.

- Detener la emisión de VLC.

- Detener herramienta generación de tráfico.

- Realizar el seguimiento del flujo RTP mediante la herramienta Stream Analysis de wireshark.

- Extraer los datos necesarios para hacer la comparación de los escenarios.

Las emisiones de video se realizaron con VLC. Una de las posibilidades de emisión de streaming en VLC es mediante el protocolo HTTP, que se sustenta sobre el protocolo de transporte TCP. Sin embargo como se sabe, TCP es orientado a la conexión, por lo cual agrega retardos indeseables en una transmisión de contenido en tiempo real. Por esto se hace uso de RTP, sustentado en UDP y que permite un control de la transmisión en tiempo real.

En Wireshark es posible hacer un seguimiento de un flujo RTP mediante la opción Stream Analysis del menú Telephony, RTP. Para las pruebas realizadas en este proyecto la única configuración relevante en Wireshark es hacer un filtrado de paquetes por número de puerto, con el fin de reducir los paquetes que se deben capturar aunque la herramienta Stream Analysis permite hacer seguimiento de flujos RTP entre diferentes tipos de tráfico. El número de puerto que se utilizo para la emisión de streaming fue el 5004, que viene configurado por defecto en VLC para RTP. La figura 5 muestra la captura de los paquetes RTP con WireShark.

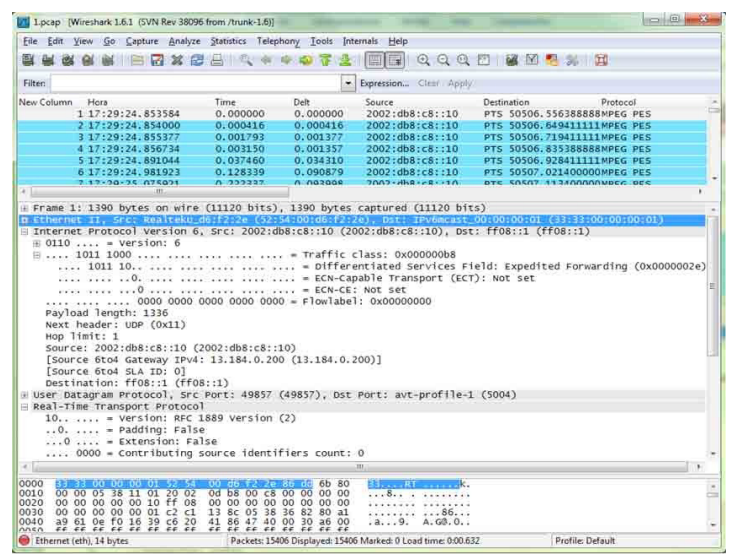

Figura 5. Captura de paquetes RTP.

\section{RESULTADOS}

Al finalizar este trabajo, se tiene un mundo virtual basado en la facultad de Ingeniería de la Universidad Distrital, alojado en un servidor con el cual se realizaron pruebas de Video Streaming utilizando las texturas de OPENSIM. Se tienen además objetos vinculados con la plataforma Moodle instalada en el mismo servidor. Los resultados se observan en las figuras 6 y 7 .

Aunque inicialmente se planteó este ambiente virtual como plataforma base para el acceso a los contenidos del repositorio, se encontraron las siguientes limitaciones:

- La reproducción de videos por medio de las texturas de OPENSIM no se hace por medio de streaming. Un video alojado en un servidor al que se apunta desde visor del lado del cliente es primero descargado al host de este último y luego es reproducido, lo cual no puede ser considerado streaming.

- El reproductor del que hace uso OPENSIM para lo anterior es QuickTime player (reproductor con licencia freeware de código cerrado), lo cual limita el formato que pueden tener los videos y los usos que se le pueden dar a los proyectos implementados.

- Ya que las texturas pueden permitir la visualización de páginas Web por medio de los navegadores que traen los visores de OPENSIM y Second Life, se tiene una alternativa al poder reproducir contenidos flash mediante streaming, aunque la capacidad de reproducir este tipo de contenidos está limitada a las funciones del navegador del visor. Hasta el momento solo el visor oficial de Second Life tiene un navegador web interno que soporta contenidos flash.

- La reproducción en OPENSIM para ninguno de los dos casos mencionados permite tener controles de reproducción, por lo cual no se puede avanzar o retroceder en el video. Únicamente se puede reproducir desde el principio, detener el video y controlar el volumen del metaverso. 


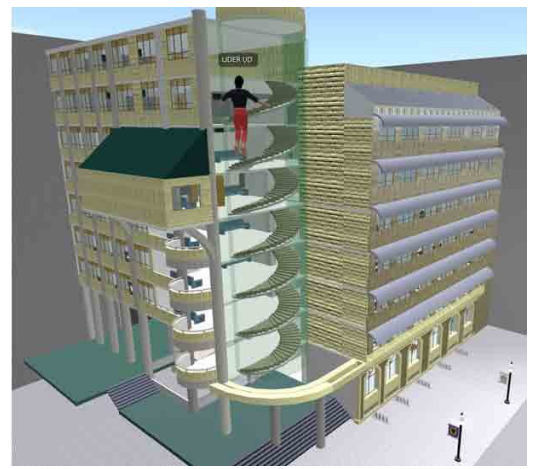

Figura 6. Metaverso Universidad Distrital.

Dadas las limitaciones se optó por la solución de desarrollar una página Web para el repositorio de video, teniendo en cuenta que el contenido Web está soportado por algunos visores de OPENSIM y para algunos tipos de video. La página web se desarrolló en HTML, y para la reproducción de contenidos por medio de navegadores se utilizó la etiqueta < video > del nuevo estándar HTML5, y el formato .webm, soporta- do nativamente por los últimos navegadores (sin necesidad de plugins). Para la reproducción en OPENSIM y antiguos navegadores se utiliza un reproductor flash embebido en el código HTML. La figura 7 muestra la página Web.

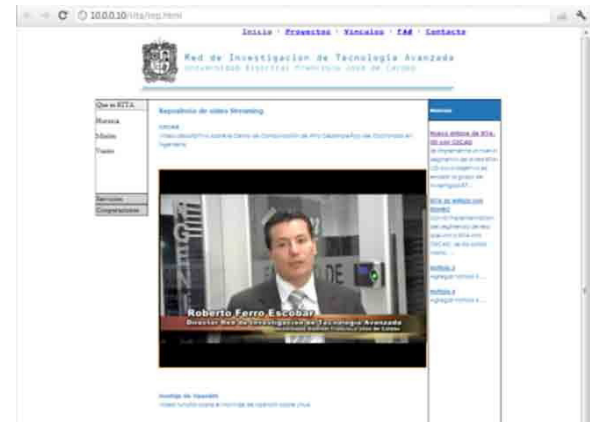

Figura 7. Página Web repositorio de video streaming.

Se realizaron transmisiones de tres tipos de video, con y sin transcodificación analizando el impacto de las emisiones sobre el servidor. La tabla 2 condensa los resultados obtenidos.

Tabla 2. Pruebas de utilización del canal durante las emisiones.

\begin{tabular}{|c|c|c|c|c|c|c|c|c|}
\hline & Libre & VNC & $\begin{array}{c}\text { VIDE0 1 } \\
\text { sin trcd }\end{array}$ & $\begin{array}{c}\text { VIDEO 2 } \\
\text { sin trcd }\end{array}$ & $\begin{array}{c}\text { VIDE0 3 } \\
\text { sin trcd }\end{array}$ & $\begin{array}{c}\text { VIDE0 1 } \\
\text { con trcd }\end{array}$ & $\begin{array}{c}\text { VIDEO 2 } \\
\text { con trcd }\end{array}$ & $\begin{array}{c}\text { VIDE0 3 } \\
\text { con trcd }\end{array}$ \\
\hline CPU & $1,56 \%$ & $10,54 \%$ & $10,89 \%$ & $15,51 \%$ & $16,54 \%$ & $57,45 \%$ & $96,54 \%$ & $97,90 \%$ \\
\hline Memoria & $8,11 \%$ & $7,09 \%$ & $7,19 \%$ & $7,54 \%$ & $7,64 \%$ & $8,78 \%$ & $10,26 \%$ & $18,03 \%$ \\
\hline Red & $0,00 \%$ & $44,69 \%$ & $10,82 \%$ & $49,73 \%$ & $100,00 \%$ & $10,75 \%$ & $19,78 \%$ & $27,25 \%$ \\
\hline
\end{tabular}

Se resalta que en la transmisión de los videos 2 y 3 con transcodificación, la sobrecarga en el servidor no permite que se pongan todos los paquetes en la red, esto explica el bajo porcentaje de utilización de la red y se traduce en una pésima reproducción del lado del cliente.

En la tabla 3 se muestran los parámetros obtenidos de las mediciones hechas sobre los flujos de datos de media streaming generados en la red RITA-UD antes de la implementación del enlace para el video 2. Para ello se utilizó un computador personal conectado a la red inalámbrica del CECAD, a una distancia de unos 7 metros aproximadamente, lo cual se refleja en un $60 \%$ a $80 \%$ de intensidad de la señal inalámbrica recibida en el host de acceso. Además se uso Iperf como herramienta de generación de tráfico para congestionar el canal.

Se realizaron pruebas de QoS desde el grupo LIDER para medir los cambios introducidos por el enlace para el video 2. Se obtuvieron los resultados que se muestran en la tabla 4. 
Tabla 3. Mediciones de QoS antes del enlace.

\begin{tabular}{|c|c|c|c|c|c|c|c|}
\hline \multicolumn{2}{|c|}{ Trafico canal } & \multicolumn{3}{c|}{ Canal libre } & \multicolumn{3}{c|}{ Congestionado } \\
\hline \multicolumn{2}{|c|}{ Traff Class } & BE & AF22 & EF & BE & AF22 & EF \\
\hline \multirow{3}{*}{ Bitrate } & Dur (s) & 59,76 & 59,12 & 59,54 & 59,87 & 59,72 & 59,04 \\
\cline { 2 - 8 } & Env & 258,77 & 259,57 & 270,82 & 255,64 & 260,27 & 261,60 \\
\hline \multirow{3}{*}{ \# de paquetes } & Env & 15464,00 & 15346,00 & 16184,00 & 15277,00 & 15554,00 & 15633,00 \\
\cline { 2 - 8 } & Rec & 11029,00 & 12864,00 & 14474,00 & 13440,00 & 13998,00 & 14264,00 \\
\cline { 2 - 8 } & \%Loss & $28,68 \%$ & $16,17 \%$ & $10,57 \%$ & $12,02 \%$ & $10,00 \%$ & $8,76 \%$ \\
\hline \multirow{3}{*}{ Secuencia } & \# Err & 339,00 & 446,00 & 239,00 & 475,00 & 772,00 & 611,00 \\
\cline { 2 - 8 } & Max loss & 145,00 & 156,00 & 107,00 & 61,00 & 54,00 & 42,00 \\
\hline \multirow{3}{*}{ Delta (ms) } & Max & 564,32 & 386,00 & 82,07 & 179,26 & 191,92 & 144,43 \\
\cline { 2 - 8 } & Prom & 5,41 & 4,85 & 4,02 & 4,45 & 4,26 & 4,14 \\
\hline \multirow{3}{*}{ Jitter (ms) } & Max & 51,64 & 42,56 & 37,90 & 19,45 & 18,77 & 18,75 \\
\cline { 2 - 8 } & Prom & 5,97 & 4,78 & 3,39 & 5,61 & 4,85 & 4,97 \\
\hline \multirow{3}{*}{ Skew (ms) } & Min & $-1570,04$ & $-365,00$ & $-992,96$ & $-389,89$ & $-304,39$ & $-256,52$ \\
\cline { 2 - 8 } & Max & 154,27 & 173,00 & 270,07 & 21,98 & 15,53 & 91,74 \\
\cline { 2 - 8 } & Prom & 31,75 & $-45,54$ & 220,16 & $-61,26$ & $-36,77$ & 25,88 \\
\hline & Min & 10,85 & 10,85 & 10,85 & 10,85 & 10,85 & 10,85 \\
\cline { 2 - 8 } & Max & 22704,86 & 74786,11 & 74786,11 & 25449,41 & 41450,21 & 46668,10 \\
\cline { 2 - 8 } & Prom & 13487,60 & 33546,78 & 43248,41 & 8032,54 & 23299,17 & 26945,86 \\
\hline
\end{tabular}

Tabla 4. Mediciones de QoS después del enlace.

\begin{tabular}{|c|c|c|c|c|c|c|c|}
\hline \multicolumn{2}{|c|}{ Traff Class } & \multicolumn{3}{c|}{ Canal libre } & \multicolumn{3}{c|}{ Congestionado } \\
\hline \multirow{2}{*}{ Modificación } & BE & AF22 & EF & BE & AF22 & EF \\
\hline \multirow{3}{*}{ Bitrate } & Dur(s) & 29,39 & 37 & 30,34 & 33,73 & 32,38 & 30,34 \\
\cline { 2 - 8 } & Pack/s & 563,865 & 555,784 & 564,403 & 535,073 & 545,862 & 546,243 \\
\hline \multirow{4}{*}{ \# de paquetes } & Env & 16572 & 20564 & 17124 & 18048 & 17675 & 16573 \\
\cline { 2 - 8 } & Rec & 15121 & 17582 & 15406 & 13059 & 13888 & 14268 \\
\cline { 2 - 8 } & \%Loss & $8,76 \%$ & $14,50 \%$ & $10,03 \%$ & $27,64 \%$ & $21,43 \%$ & $13,91 \%$ \\
\hline \multirow{3}{*}{ Secuencia } & \# Err & 776 & 1965 & 962 & 3299 & 9223 & 1496 \\
\cline { 2 - 8 } & Max loss & 27 & 50 & 46 & 128 & 24 & 36 \\
\hline \multirow{3}{*}{ Delta (ms) } & Max & 109,43 & 270,77 & 141,91 & 669,56 & 679,28 & 632,27 \\
\cline { 2 - 8 } & Prom & 1,94349 & 2,10435 & 1,96934 & 2,58261 & 2,33133 & 2,12615 \\
\hline \multirow{3}{*}{ Jitter (ms) } & Max & 16,94 & 16,96 & 17,78 & 14695,3 & 12436,9 & 9203,71 \\
\cline { 2 - 8 } & Prom & 1,68665 & 1,70397 & 1,6399 & 20,2266 & 16,5313 & 12,277 \\
\hline \multirow{3}{*}{ Skew (ms) } & Min & $-681,05$ & $-729,21$ & $-694,92$ & 0 & 0 & 0 \\
\cline { 2 - 8 } & Max & 372,73 & 386,78 & 372,9 & 235201 & 199066 & 147341 \\
\cline { 2 - 8 } & Prom & 26,7101 & 52,6885 & $-48,354$ & 234469 & 198330 & 146842 \\
\hline \multirow{3}{*}{ IP BW (kbps) } & Min & 10,85 & 10,85 & 10,85 & 10,85 & 10,85 & 10,85 \\
\cline { 2 - 8 } & Max & 161744 & 188560 & 164347 & 132671 & 148563 & 151655 \\
\cline { 2 - 8 } & Prom & 81589,4 & 94597,3 & 83026,5 & 69642 & 74802,1 & 76902,7 \\
\hline
\end{tabular}


Analizando la tabla 3 y tabla 4 se obtuvieron los siguientes resultados:

- Aunque se encontraron mejores parámetros de QoS para el flujo de datos marcado con el DSCP Expedited Forwarding bajo un escenario de tráfico con IPERF se hicieron otras pruebas sobre el video de menor calidad, encontrando que el flujo de datos no se ve afectado de manera considerable por los diferentes tipos de tráfico alterno debido a su baja tasa de bits.

- En cuanto al video de mayor calidad, se tienen claramente mejores resultados para el video sin tráfico alterno. Sin embargo no se tiene una diferencia considerable entre las diferentes clases de tráfico.

\section{CONCLUSIONES}

- Con la construcción del metaverso de la sede de ingeniería de la Universidad Distrital, se comprobó la facilidad que presenta OPENSIM para el montaje de mundos virtuales funcionales. Sin embargo, la forma en que se reproducen videos por medio de las texturas y las características del navegador Web que traen los visores limita el tipo de streaming que puede hacerse dentro de estos. Se recomienda hacer grupos de trabajo que experimenten con las funciones avanzadas de OPENSIM.

- Si bien el uso de video streaming dentro de los mundos virtuales mejora la experiencia

Referencias Bibliográficas

[1] B. Juan, C. Ernesto, Prototipo de un servicio de media streaming sobre ipv6 para la red de tecnología avanzada RITAUD con acceso desde la red metropolitana rumbo, mediante software libre y el modelo TMN de la UIT-T. Bogotá, 2011, 168 h. Trabajo de grado (Ingeniero Electrónico). Universidad Distrital Francisco José de Caldas: Facultad de Ingeniería.

[2] A. Víctor, A. Diego, Diseño e implementación de la infraestructura de de aprendizaje en un entorno educativo virtual, por su limitada capacidad actual para reproducción de diferentes tipos de streaming y formatos, y el control que se puede tener sobre los mismos por parte del cliente, estos no son óptimos como plataformas de acceso a un repositorio de video.

- Para enlaces de red en los que el ancho de banda sea un factor crítico debido al volumen de datos a transmitir, existe un número máximo de repeticiones que condiciona a calidad del enlace. En este caso, para la transmisión de un video de mediana calidad entre el CECAD y el grupo LIDER, no es posible tener más de dos repeticiones.

- A pesar de que la solución del enlace híbrido no ofrece un ancho de banda alto en comparación con una solución cableada, es suficiente para la transmisión de un video de mediana calidad, transmisión que en multicast permite la conexión de múltiples usuarios delegando la responsabilidad de ancho de banda y distribución al extremo receptor del enlace.

- Contrastando los resultados obtenidos antes y después del enlace se concluyó que antes del enlace una modificación del campo DSCP para una transmisión de video de mediana o alta calidad en condiciones normales de uso de la red no ofrece ventajas frente a un tráfico no modificado, ya que el ancho de banda por usuario es suficiente para soportar los múltiples flujos de datos que se dan paralelamente.

la red interna Del Centro de Computación de Alto Desempeño CECAD de la Universidad Distrital mediante el modelo TMN de la UIT-T. Bogotá, 2011, 135 h. Trabajo de grado (Ingeniero Electrónico). Universidad Distrital Francisco José de Caldas: Facultad de Ingeniería.

[3] M. Carlos, D. Carlos, Diseño de un prototipo de conexión de la red de tecnología avanzada TIRA-UD con la red metropolitana RUMBO bajo Iv6 
mediante software libre y el modelo TMN de la UIT-T. Bogotá, 2009, 145 h. Trabajo de grado (Ingeniero Electrónico). Universidad Distrital Francisco José de Caldas: Facultad de Ingeniería.

[4] AXIS. Las redes IP: Conceptos básicos. [En línea], consultado en Agosto 3 del 2011, disponible en: http://www. casadomo.com/casadomo/biblioteca/ axis_las_redes_ip.pdf

[5] RUMBO. Rumbo - Renata - Clara: Un Camino hacia la e-Ciencia: Redes Académicas Avanzadas de Alta Velocidad. [En línea], consultado en Julio 21 del 2011, disponible en: http://www.rumbo. edu.co/file.php/1/documentos/Informa RUMBO.pdf

[6] E. Escobar, M. Flores, M. Medina, M. Rodríguez, S. Olivia, Tecnologías de la información y la comunicación, aplicadas a la enseñanza y el aprendizaje: UNLAR, 2009. $313 \mathrm{p}$.

[7] VC. Esains, Contenidos para e-Learning. Learning Review España, año 2, $\mathrm{N}^{\mathrm{o}} 6$, Abril -Mayo -Junio 2009.

[8] Opensimulator Project, OpenSim documentation. [En línea], consultado en Septiembre 21 del 2011, disponible en: http://www.opensim.org

[9] H. Roberto, F. Carlos y B. Pilar, Fundamentos de metodología de la investigación, Cuarta edición, México: McGraw Hill/Interamericana de México, 2007.

[10] P. McLean, Exploring time capsule: Theoretical speed vs practical throughput. [En línea], consultado en Junio 28 del 201, disponible en: http://appleinsider. com/articles/08/03/28/exploring time capsule_theoretical_speed_vs_practical_ throughput.html

[11] RFC 6052, IPv6 addressing of IPv4/IPv6 translators, [En línea], consultado en octubre 20 del 2011, disponible en: http:// tools.ietf.org/html/ $/ \mathrm{fc} 6052$

[12] RFC-2460, [En línea], consultado en Marzo 2 del 2011, disponible en: http:// tools.ietf.org/html/rfc2460

VideoLAN. [En línea], consultado en Octubre 22 del 2011, disponible en: http:// wiki.videolan.org/VideoLAN_Sites 